\title{
A Review on Effect of Selenium Enriched Tea as Antioxidant
}

\author{
Irfana, Deepika P*, Shiji kumar P. S, Sirajudheen M. K, Sherin A
}

Jamia Salafiya Pharmacy College, Pulikkal, Malappuram, Kerala, India

This present review on antioxidant property of selenium infused tea focused on various assay techniques to demonstrate its high antioxidant efficacy compared to normal tea and its benefits in relation to various medical conditions. From the different assay techniques, it is found that tea rich in selenium has a higher antioxidant property than regular tea. This is mainly due to the ability of selenium to act as antioxidant and anti-free radical. In addition to this selenium infused tea also exhibits various antioxidant mechanisms such as glutathione peroxidase like activity, in oxidative stress, cancer prevention, etc. Since selenium is an important component of glutathione peroxidase, it can eradicate various stress reactions as well as the formation of free radicals by its radical scavenging properties. Because of this property of selenium, selenium-infused tea is more effective in various types of cancer compared to normal green tea.

Keywords: Selenium, green tea, antioxidant.

Copyright @ 2020: This is an open-access article distributed under the terms of the Creative Commons Attribution license which permits unrestricted use, distribution, and reproduction in any medium for non-commercial use (NonCommercial, or CC-BY-NC) provided the original author and source are credited.

\section{INTRODUCTION}

Tea is one of the commonly used beverage with Caffeine, polyphenol, theanine as major constituents. It exhibits a wide range of health benefits especially in chronic diseases like cancer, inflammatory diseases as a result of its antioxidant, anti-mutagenic, and anticancer effects. It is available in various formsblack, white, green tea depending upon its method of production. High phenol content of tea is responsible for its antioxidant activity. Various assay techniques are employed to evaluate antioxidant property of green tea which shows that free radical scavenging property, ferrous ion chelating activity are criterias for its increased antioxidant property.

Selenium is a trace element or micronutrient that have a potential role in human health as antigenotoxic, chemopreventive, and in metabolic pathways like thyroid hormone metabolism, antioxidant defence systems. Organic form of selenium has greater bioavailability and least toxicity than inorganic form. Inadequate amount of selenium in the diet may lead to serious health problems like cardiovascular diseases, infertility, arthritis (pain, swelling in joints) due to oxidative damage to cell. Selenium was able to remove free radicals in the body which is produced as a byproduct of oxygen metabolism. Harvesting of tea is mainly done at summer tea-producing season in order to obtain good quality. Since selenium can improve the qualities (bitterness, astringent taste, antioxidant property) of tea, it is incorporated either through fertilizers or by directly spraying it on to tea leaves.

This review illustrates the antioxidant property of selenium-enriched tea by various assay techniques like 1,1-diphenyl-2-picrylhydrazyl (DPPH)radicals, cupric reducing antioxidant capacity (CUPRAC) and Folin Ciocalteu (FC) assays. From various results, it is clearly shown that the antioxidant capacity of seleniumenriched tea is higher than regular tea. This review mainly aimed at the antioxidant property, biological activities and therapeautic activities of selenium enriched tea.

\section{REVIEW}

Jaun $\mathrm{Xu}$ et al., studied the influence of selenium on vitamin c, polyphenol contents of tea and determined whether tea enriched with selenium has higher antioxidant property or not. Green tea samples were prepared in summer tea producing season. Selenium is incorporated into tea by fertilizing soil or by foliar application. Antioxidant property was evaluated by lard oxidation and linoleic acid oxidation. In lard oxidation, mixture was prepared by taking equal concentration of butylated hydroxy toluene (BHT) and tea extract which is then mixed with lard and maintained at specific temperature. Then peroxide value 
is determined. A higher peroxide value indicates weak antioxidant property. Linoleic acid oxidation is undergone by mixing equal amount of tea extract and linoleic acid which is then mixed with ethanol, ammonium thiocyanate and absorbance was measured, until absorbance reaches steady state. In lard oxidation a lower peroxide value is indicated for selenium enriched tea which exhibits its high antioxidant property. Likewise, In linoleic acid oxidation it exhibits lower absorbance for tea enriched with selenium. From both assays it shows higher antioxidant property for selenium enriched tea than regular tea [1].

Ines L. Martins et al., synthesised 6selenocaffeine and evaluated for its antioxidant potential. It is synthesised using Woollin's reagent which allowed an efficient conversion of caffeine into 6-selenocaffeine.Woollin's reagent is a selenium analogue that selenates caffeine by replacing oxygen atom in caffeine with selenium. Antioxidant property of 6-selenocaffeine assessed by diphenyl-Picrylhydrazyl (DPPH) exhibits decreased amount of DPPH radical than that of caffeine, which is due to marked scavenging effect of selenium in 6-selenocaffeine. On evaluating antioxidant activity upon selenation of caffeine, using the DPPH assay, indicated a mild antioxidant activity for 6-selenocaffeine, contrasting with caffeine, that exhibited no antioxidant activity under the same experiment conditions. Results shows that organo selenium compounds in Selenium rich green tea exhibits wide range of antioxidant mechanisms such as Glutathione peroxidase like activity, chelating property, inhibitory activity on peroxide [2].

Molan et al., [3] compared the antioxidant activity of China green tea and selenium rich green tea by preparing their extracts. Antioxidant property was evaluated by various assay techniques such as Ferric reducing antioxidant power assay, Diphenyl picrylhydrazyl (DPPH) assay and Ferrous ion chelating assay. Ferric reducing antioxidant power assay was based on the ability of tea extract to reduce ferric ions. For this mixture was prepared using tea extract and Ferric reducing antioxidant power (FRAP) reagent. Also, standard curve is produced for different concentration of ferrous sulphate. It shows that selenium rich green tea has high reducing activity which is an indication of antioxidant property. In DPPH assay, radical scavenging ability of sample is compared to that of control (ascorbic acid). Then, antiradical activity was measured as follows:

Antiradical activity \%= (absorbance of control incubation-absorbance of tea extract)/absorbance of control incubation $\times 100$

Ferrous ion chelating assay was based on the ability of tea extract to chelate with ferrous ion which is calculated as follows:
Chelating activity $\%=$ (absorbance of control incubation-absorbance of tea extract)/absorbance of control incubation

While examining the effect of both selenium infused and regular tea extracts on growth of lactobacillus, it was found that tea containing high proportion of selenium was more effective in improving growth of bacteria. The reason behind this was ability of selenium rich tea to act as antioxidant and as antiradical which modulate oxidative stress and create a better environment for bacterial growth [3].

Fang yu et al., compared antioxidant activity of selenium-brewed tea and regular green tea. Selenium rich green tea was prepared by foliar application of selenium on green tea and was found to contain polyphenol, proteins, polysaccharides and selenium. The antioxidant property of both forms of tea was assessed by auto-oxidation test (AAPH) and diphenyl picrylhydrazyl (DPPH) assay techniques. In AAPH technique, tea extracts are mixed with equal volume of linoleic acid and peroxidized. 2, 2' -Azobis(2amidinopropane)dihydrochloride (AAPH)is added to initiate peroxidation. Degree of oxidation was determined from peroxide value, which is determined by reading absorbance. For DPPH assay, antioxidant property of tea polyphenols, polysaccharides, proteins were evaluated using DPPH radicals. The decrease in absorbance was measured until reaction reaches steady state. Then EC50 value was determined which indicates amount of antioxidant necessary to lower the level of DPPH. In both tests, selenium rich green tea have higher antioxidant property compared to regular tea. It shows that selenium in combination with tea polyphenols and polysaccharides has high antioxidant activity compared with normal tea [4].

Feng Li et al., prepared aqueous and ethanolic extracts of green tea rich in selenium and determined degree of oxidation by diphenyl picrylhydrazyl (DPPH) and ferric thiocyanate (FTC) assays. Radical scavenging ability of tea extract was determined by measuring absorbance of aqueous and ethanolic extracts. Decrease in absorbance is measured until absorption reaches steady state. As antioxidants react with DPPH, they decrease amount of DPPH and absorbance in response to DPPH decreases. Since, ethanolic green tea extracts rich in selenium have low absorbance measured, it indicate their high antioxidant property. In FTC method, antioxidant properties of aqueous and ethanolic extracts of selenium rich green tea was evaluated by linoleic acid system and determined amount of peroxide formed. A decrease in absorbance measured and low concentration of peroxide formed leads to high antioxidant property. Since, ethanolic extracts of selenium rich green tea delays oxidation of linoleic acid, amount of peroxide formed reduced, which shows low absorbance and thereby high antioxidant property. This is due to high proportion of 
selenium in selenium rich green tea which is attributed to its antioxidant property [5].

Quihi $\mathrm{Hu}$ et al., examined the physiological function of selenium infused tea fertilized with sodium selenite and its effect on animals. Initially, experiments with selenium rich green tea were carried out and subsequent experiments were carried out on animals such as rats. The use of the extract was determined after feeding rats for few weeks with tea and subsequent determination of glutathione peroxidase activity, malondialdehyde content (MDC) and selenium content in blood and liver. Selenium content analyzed using statistical methods. Selenium content varies among natural tea rich in selenium and tea infused with selenium. High level of organic selenium was found with tea fertilized with selenium in soil. It combines with various proteins in tea and exhibit high biological activity. Circulatory and hepatic glutathione peroxidase activity was increased in group of rats fed with tea infused with selenium. Significant difference in activity of glutathione peroxidase between two forms of tea suggest that tea fortified with selenium was more effective than normal tea. The MDA content, which is a measure of lipid oxidation found decreased in rats fed on tea rich in selenium which is due to its antioxidant potential. It thus shows the ability of selenium to act as antioxidant [6].

Aleksandra Sentkowska et al., investigated antioxidant activity of selenium enriched tea by conducting various assay techniques like DPPH assay which is used to evaluate radical scavenging property of green tea extract, Cupric reducing antioxidant capacity (CUPRAC) of tea and by Folin-Ciocalteau (FC) assay which determines total phenolic content. The various species of selenium available are Selenium methionine (SeMet), Se(1V), Se(VI) and methylselenocysteine (MeSeCys). Se (IV) exhibited lowest antioxidant properties compared to other selenium compounds. The highest reducing power was for SeMet and high DPPH radical scavenging ability is for MeSeCys. He has also studied the influence of different species of selenium on green tea as well as black tea and found that selenoaminoacid in black tea decreased its antioxidant potency while not in green tea [7].

Sunisa et al., examined ability of selenium, a trace element, as an antioxidant system. Bioenrichment with selenium can improve the phenol content of tea, which is considered to be its major component. Reactive oxygen species, free radicals are factors for cell injury. In order to eradicate them there are enzymatic (GSH-Peroxidase, Catalase, peroxidase) as well as non-enzymatic (ascorbic acid, phenolic compounds) antioxidants in plants. Since, selenium is an important component of Glutathione Peroxidase enzyme, it helps in various kinds of stress reactions by improving Glutathione peroxidase level in plants. Improved antioxidant property in plants by selenium was measured by various assay techniques. In order to demonstrate the effect of GSH-Peroxidase in-vivo, experiments were performed in rats. In vitro studies on ethanolic extract of green tea shown that it inhibit growth of cancer cells which is attributed to the ability of selenium to increase antioxidant effectiveness. Only appropriate amount of selenium is beneficial as high dose may exert toxic effects [8].

Huajia Li et al., evaluated antioxidant efficacy of superficial sized selenium-rich green tea and regular tea. It is determined by DPPH and FTC assays. The inhibition of free radical was measured until absorbance reaches steady state. A high inhibition rate indicate high antioxidant potential as shown by Selenium rich green tea. In FTC assay concentration of peroxide formed is determined. High inhibition of linoleic acid results in low absorbance, which indicate low concentration of peroxide formed and high antioxidant property, which is exhibited by selenium rich green tea. Two tests shows that selenium rich green tea has high antioxidant property compared to normal tea, both having same particle size. It is due to the ability of selenium to act as antioxidant [9].

Noor Hazarina Nordin et al., compared antioxidant activities of selenium rich black tea and regular black tea by determining total phenolic content. Initially extracts of regular black tea and selenium rich black tea was prepared. Phenolic content have greater influence on antioxidant activity by inhibiting lipid oxidation, by free radical scavenging. Total phenolic content of tea extract was determined by FC assay and it is determined from calibration curve of Gallic acid Results showed that regular black tea with a high phenol content than black tea rich in selenium. On evaluating antioxidant potency of tea extract, high ferric reducing power, high DPPH scavenging abilities are exhibited by regular black tea than selenium rich black tea [10].

Fangmei Yang et al., Selenium and green tea have been shown to have potential antitumor effects. Anti cancer property of selenium-enriched green tea extract (Se-TE) in a Kunming mice model transplanted with human hepatoma cells HepG2 was investigated. Mice were divided to 8 groups with 10 mice in each group, after tumor cell inoculation. One of the group preserved as control which is fed with only water, whereas the remaining groups received regular green tea extract (RT), Selenium infused tea extract (Se-TE). Tumour growth suppression in mice fed with these agents compared with that of control which exhibits higher growth suppressipon in mice fed with Se-TE. The results suggest that the antitumor function of SeTEs may be attributed to the oxidative stress induced by selenium and green tea components [11]. 
Yuanfeng Wang et al., examined antioxidant activities of selenium enriched tea. Selenium polysaccharides from selenium enriched tea leaves were isolated and purified. Antioxidant property was assessed by DPPH radical scavenging activity, Scavenging effect on superoxide radical assay. In DPPH scavenging assay absorbance of sample is measured and compared with positive control (ascorbic acid) and ethyl alcohol (negative control). Scavenging effect was calculated as follows: Scavenging rate $(\%)=$ $[1-(\mathrm{Ai}-\mathrm{Aj}) / \mathrm{Ao}] \times 100$. Result shows selenium enriched tea with improved radical scavenging activity than control. In superoxide anion scavenging assay, it also exhibits better scavenging activity for selenium infused tea than control. From this two assays it shows improved antioxidant property for selenium rich tea than regular tea [12].

\section{REFERENCES}

1. Xu J, Zhu S, Yang F, Cheng L, Hu Y, Pan G, Hu Q. The influence of selenium on the antioxidant activity of green tea. Journal of the Science of Food and Agriculture. 2003 Apr;83(5):451-5.

2. Martins IL, Miranda JP, Oliveira NG, Fernandes AS, Gonçalves S, Antunes AM. Synthesis and biological activity of 6-selenocaffeine: potential modulator of chemotherapeutic drugs in breast cancer cells. Molecules. 2013 May;18(5):5251-64.

3. Molan AL, Flanagan J, Wei W, Moughan PJ. Selenium-containing green tea has higher antioxidant and prebiotic activities than regular green tea. Food Chemistry. 2009 Jun 1;114(3):829-35.

4. $\mathrm{Yu} \mathrm{F}$, Sheng J, Xu J, An X, Hu Q. Antioxidant activities of crude tea polyphenols, polysaccharides and proteins of selenium-enriched tea and regular green tea. European Food Research and Technology. 2007 Sep 1;225(5-6):843-848.
5. Li F, Wang F, Yu F, Fang Y, Xin Z, Yang F, Xu J, Zhao L, Hu Q. In vitro antioxidant and anticancer activities of ethanolic extract of selenium-enriched green tea. Food Chemistry. 2008 Nov 1;111(1):165-70.

6. Hu Q, Pan G, An X, Ding R. Physiological function of Se- enriched tea fertilised with sodium selenite and naturally high- Se tea in rats. Journal of the Science of Food and Agriculture. 2001 Jan 15;81(2):202-4.

7. Sentkowska A, Pyrzyńska K. Investigation of antioxidant activity of selenium compounds and their mixtures with tea polyphenols. Molecular biology reports. 2019 Jun 1;46(3):3019-24.

8. Chomchan R, Siripongvutikorn S, Puttarak P. Selenium bio-fortification: an alternative to improve phytochemicals and bioactivities of plant foods. Functional foods in health and disease. 2017 Apr 30;7(4):263-79.

9. Li H, Li F, Yang F, Fang Y, Xin Z, Zhao L, Hu Q. Size effect of Se-enriched green tea particles on in vitro antioxidant and antitumor activities. Journal of agricultural and food chemistry. 2008 Jun 25;56(12):4529-33.

10. Nordin NH, Molan AL, Chua W, Kruger MC. Total phenolic contents and antioxidant activities of selenium-rich black tea versus regular black tea. American journal of Life Science Researches, 2017; 5(2):40-50.

11. $\mathrm{Xu} \mathrm{J,} \mathrm{Yang} \mathrm{F,} \mathrm{An} \mathrm{X}, \mathrm{Hu}$ Q. Anticarcinogenic activity of selenium-enriched green tea extracts in vivo. Journal of agricultural and food chemistry. 2007 Jun 27;55(13):5349-53.

12. Wang Y, Li Y, Liu Y, Chen X, Wei X. Extraction, characterization and antioxidant activities of Seenriched tea polysaccharides. International journal of biological macromolecules. 2015 Jun 1;77:7684. 